\title{
Enhanced Crystallinity and Antibacterial of PHBV Scaffolds Incorporated with Zinc Oxide
}

\author{
Cijun Shuai $\mathbb{D},{ }^{1,2,3}$ Chen Wang, ${ }^{1}$ Fangwei Qi $\mathbb{D},{ }^{1}$ Shuping Peng $\mathbb{D},{ }^{4,5}$ Wenjing Yang, \\ Chongxian He, ${ }^{1}$ Guoyong Wang, ${ }^{1}$ and Guowen Qian ${ }^{1}$ \\ ${ }^{1}$ Institute of Bioadditive Manufacturing, Jiangxi University of Science and Technology, Nanchang 330013, China \\ ${ }^{2}$ State Key Laboratory of High Performance Complex Manufacturing, Central South University, Changsha 410083, China \\ ${ }^{3}$ Shenzhen Institute of Information Technology, Shenzhen 518172, China \\ ${ }^{4}$ NHC Key Laboratory of Carcinogenesis, School of basic Medical Science, Central South University, Changsha, Hunan 410013, China \\ ${ }^{5}$ School of Energy and Machinery Engineering, Jiangxi University of Science and Technology, Nanchang 330013, China
}

Correspondence should be addressed to Fangwei Qi; qfw@jxust.edu.cn and Shuping Peng; shuping@csu.edu.cn

Received 29 May 2020; Accepted 29 June 2020; Published 8 July 2020

Academic Editor: Xiaoming Li

Copyright (C) 2020 Cijun Shuai et al. This is an open access article distributed under the Creative Commons Attribution License, which permits unrestricted use, distribution, and reproduction in any medium, provided the original work is properly cited.

Poly(3-hydroxybutyrate-co-3-hydroxyvalerate) (PHBV) has a great potential in bone repair, but unfortunately, the poor mechanical properties limit its further application. In this work, zinc oxide $(\mathrm{ZnO})$ nanoparticles were incorporated into PHBV porous scaffold fabricated by selective laser sintering technique. It was because $\mathrm{ZnO}$ nanoparticles could provide nucleating sites for the orderly stacking of polymer chains, thereby enhancing the crystallinity of PHBV. It was well known that the mechanical properties of PHBV scaffold could be enhanced with the increase of crystallinity. More significantly, the released $\mathrm{Zn}^{2+}$ would combine negatively charged cell membranes of bacterial through electrostatic interaction and consequently destructed the protein structure and resulted in the death of bacterial, which was highly desired in reducing the risk of implant infection. Results indicated that the relative crystallinity of scaffold with $3 \mathrm{wt} . \% \mathrm{ZnO}$ increased remarkably from $38 \%$ to $64 \%$ compared to pure PHBV scaffold, which effectively enhanced the compression strength and modulus by $56 \%$ and $51.5 \%$, respectively. Moreover, the scaffold had a favorable antibacterial activity. Cell culture experiments proved that the scaffold could promote the cell behaviors. The positive results demonstrated the scaffold may serve as a potential replacement in bone repair.

\section{Introduction}

The large bone defects resulted from trauma, infection, and tumor are commonly difficult to heal by their own ability, which seriously affects the patients' living quality and health level [1]. Artificial bone scaffolds, which enable to provide a mechanical and physiological support to cells for in vitro tissue regeneration and/or in vivo implantation, are considered to be a promising alternative in the treatment of large bone defects $[2,3]$. Among various biomaterials, poly(3-hydroxybutyrate-co-3-hydroxyvalerate) (PHBV) has received considerable attention because of its favorable biocompatibility and biodegradability. It can degrade in vivo to hydroxybutyric, which is a normal component of human blood [4]. However, insufficient mechanical performance has limited its further application in bone repair.

It is well established that the mechanical property of PHBV is positively related to its crystallinity [5]. Introducing nanofillers is considered to be an effective countermeasure to improve the crystallinity [6]. For example, Jun et al. [7] have found that the crystallinity of PHBV was greatly improved with the incorporation of carbon nanotubes. Kouhi et al. [8] fabricated PHBV/hydroxyapatite/bredigite scaffolds and found that the incorporation of nanoparticles increased the crystallinity of PHBV matrix and subsequently enhanced the Young's modulus and ultimate strength. Zhang et al. [9] prepared PHBV/cellulose nanocrystals/silver nanocomposites by using solution casting. It was found that the composites showed higher 
crystallinity and smaller average crystallite size compared to PHBV. Meanwhile, the tensile strength and modulus of the composites were greatly increased by $97.6 \%$ and $231 \%$, respectively.

Among various nanofillers, zinc oxide $(\mathrm{ZnO})$ nanoparticles possess good biocompatibility, which has been approved as a biomedical material by Food and Drug Administration (FDA) [10]. More interestingly, $\mathrm{ZnO}$ has a favorable antibacterial ability against a wide a range of bacteria. It can release zinc ions $\left(\mathrm{Zn}^{2+}\right)$ to combine with the negatively charged cell membranes of bacterial through electrostatic interaction and consequently destructed the protein structure and resulted in the death of bacterial, which was highly desired in reducing the risk of implant infection [11]. Furthermore, it can also generate reactive oxygen species (ROS) under the irradiation of ultraviolet light to damage the cell membranes of bacterial $[12,13]$. Moreover, $\mathrm{Zn}$ is an important trace element in the human body and participates in various metabolic processes [14]. It has been reported that an appropriate amount of $\mathrm{Zn}$ can promote bone formation while inhibiting bone resorption.

Although different fabrication methods including solvent casting, particle-leaching, and foam replication have been developed to manufacture bone scaffolds with desirable properties, these techniques have great difficulty in fabricating bone scaffolds with controllable porous structure and personalized shape [15]. It is well established that the porous structure of the scaffold is conducive to the growth of cells, the transmission of nutrients, and the discharge of metabolites, whereas the personalized shape design can meet the requirements of various patients $[16,17]$. As an emerging additive manufacturing technique, selective laser sintering (SLS) can precisely construct bone scaffold with complicated structure and customized shape from 3D digital data by sequentially fusing region in a powder bed, layer-by-layer, via a computer-controlled scanning laser beam. More particularly, any powdered biomaterial that will fuse but not decompose under the irradiation of laser beam can be used to fabricate scaffold [18].

In this work, $\mathrm{PHBV} / \mathrm{ZnO}$ scaffolds were manufactured by selective laser sintering. The crystallization behaviors, mechanical performances, and antibacterial activities were systematically investigated. The relationship between the crystallization behavior and mechanical properties was discussed in depth. Moreover, in vitro cell behaviors were also explored. This work attempts to provide an effective countermeasure to simultaneously improve the mechanical property and antibacterial activity of PHBV scaffold and also supplies a strategy to overcome challenges in rapidly fabricating scaffolds with controllable pore structure.

\section{Experiment}

2.1. Materials. PHBV powders (molecular weight: $280 \mathrm{kDa}$, density: $1.25 \mathrm{~g} / \mathrm{cm}^{3}$ ) were provided by TianAn Biological Materials Co., Ltd. (Ningbo, China). ZnO nanoparticles with average size of $50 \mathrm{~nm}$ and specific surface area of $15-25 \mathrm{~m}^{2} / \mathrm{g}$ were supplied by Aladdin Reagent Co., Ltd. (Shanghai, China).
2.2. Preparation of $P H B V I Z n O$ Scaffold. The fabrication process of three-dimensional porous $\mathrm{PHBV} / \mathrm{ZnO}$ composite scaffolds is illustrated in Figure 1. In detail, a certain amount of $\mathrm{PHBV}$ and $\mathrm{ZnO}$ powders was weighted according to the designed mass ratio and then poured into a beaker containing ethanol solution. Subsequently, the mixed suspensions were subjected to mechanical agitation and ultrasonic dispersion simultaneously for $2 \mathrm{~h}$. Afterwards, the well-mixed suspensions were filtered and dried in the vacuum drying oven. Ultimately, the mixed powders could be obtained by using ball milling.

The scaffolds were manufactured by a laser 3D molding system with independent intellectual property rights. It mainly consisted of a continuous wave $\mathrm{CO}_{2}$ laser with the wavelength of $10.6 \mu \mathrm{m}$. The whole processing parameters in this study were set as follows: laser power $5 \mathrm{~W}$, scanning speed $100 \mathrm{~mm} / \mathrm{s}$, and scanning space $0.15 \mathrm{~mm}$. The threedimensional porous scaffolds could be obtained through layer-by-layer construction. For the convenience of description, PHBV scaffold with various $\mathrm{ZnO}$ contents of 1,3 , and 5 wt.\%, which were named as $\mathrm{PHBV} / 1 \mathrm{nZnO}, \mathrm{PHBV} / 3 \mathrm{nZnO}$, and $\mathrm{PHBV} / 5 \mathrm{nZnO}$, respectively.

2.3. Microstructure and Mechanical Property. The morphologies of the samples were characterized by scanning electron microscopy (SEM, Zeiss, Germany). The functional groups of the scaffolds were analyzed by the Fourier transform infrared spectroscopy (FTIR, Tianjin Gang Dong Technology Co. Ltd., China). The phase structures of the scaffolds were measured by utilizing X-ray diffractometer (XRD, Karlsruhe, Germany). The melting and crystallization performance of the scaffolds under a constant heating and cooling rate of $10^{\circ} \mathrm{C} / \mathrm{min}$ was measured by differential scanning calorimeter (DSC, TA, USA). The samples were firstly heated from 30 to $210^{\circ} \mathrm{C}$ to remove the thermal story of the PHBV and then cooled to $30^{\circ} \mathrm{C}$. Afterwards, the samples were heated to $210^{\circ} \mathrm{C}$ again. The thermal stabilities of the samples were investigated by thermogravimetric analyzer (TG, PerkinElmer, USA). The $\mathrm{Zn}^{2+}$ concentration released by scaffold in the deionized water was quantitative analyzed via inductively coupled plasma optical emission spectrometer (ICP-OES, SPECTROBLUE SOP, Germany). The compression properties of the scaffolds were measured by mechanical testing machine (CMTS5205, MTS, USA) under a deformation rate of $0.5 \mathrm{~mm} / \mathrm{s}$.

2.4. Antibacterial Activity. Before the experiment, all experimental apparatus and the samples were washed with ethyl alcohol under the ultrasound bath and subsequently sterilized with ultraviolet (UV) for $1 \mathrm{~h}$. The phosphate buffer solution (PBS) was used to dilute the bacterial suspensions and then seeded to lysogeny broth (LB) culture medium.

The Escherichia coli (E. coli, ATCC 25922) were selected to explore the antibacterial properties of the scaffolds. The antibacterial properties of the scaffolds against E. coli were quantitatively evaluated by the bacterial inhibition rate. In detail, the scaffolds with various $\mathrm{ZnO}$ contents were immersed in the Petri dish containing bacterial suspensions with a density of $1 \times 10^{6} \mathrm{CFU} / \mathrm{mL}$ and cultured for 1,4 , and 


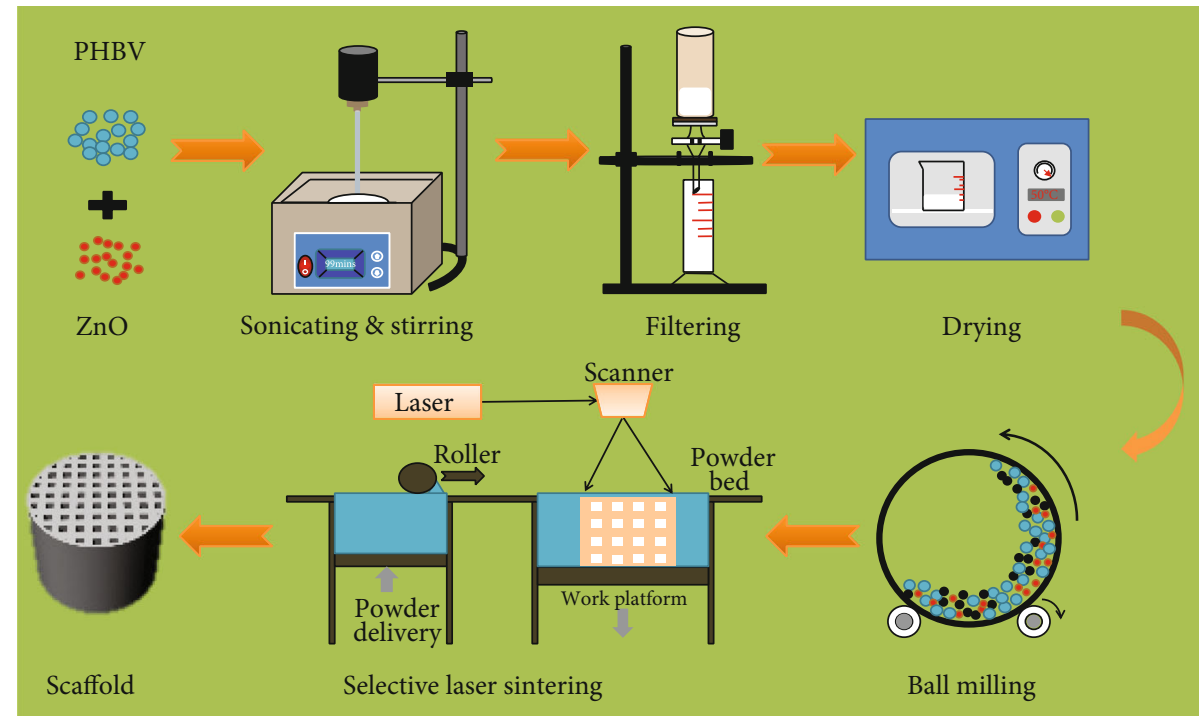

FIGURE 1: The manufacturing process of the scaffolds.

7 days at $37^{\circ} \mathrm{C}$. Then, the optical density of bacterial was also measured by a microplate reader (Beckman, USA) at $600 \mathrm{~nm}$. The antibacterial rate was calculated as the following equation:

$$
\text { Antibacterial rate }(\%)=\frac{A_{2}-A_{1}}{A_{1}} \times 100 \%
$$

where $A_{2}$ and $A_{1}$ were the optical density of the bacterial suspensions contained with and without scaffolds, respectively. Each sample was tested for three times.

The morphologies of bacterial on the scaffolds were observed by SEM. In detail, the bacterial-scaffold complexes were taken out from the Petri dish and cleaned with PBS. Afterwards, the bacterial-scaffold complexes were fixed with glutaraldehyde and dehydrated with a series of alcohol. Subsequently, the complex was dried in vacuum drying oven and finally observed by SEM.

2.5. Cell Response. The cell response of the scaffolds was evaluated by using human osteoblast-like MG-63 cells. Prior to testing, the cells were cultured in glucose DMEM containing $10 \%$ fetal bovine serum at $37^{\circ} \mathrm{C}$ under a humidified environment with $5 \% \mathrm{CO}_{2}$, and the culture medium was refreshed every two days. Before cell seeding, the scaffolds were washed with PBS and sterilized with UV for $30 \mathrm{~min}$ followed by transferring them into a 12-well dish. Subsequently, the cells were seeded on scaffolds with a density of $1 \times 10^{4} \mathrm{cell} / \mathrm{mL}$.

The cell adhesion on scaffold cultured for various periods was observed by SEM. After 1, 3, and 5 days of cultivation, the cell-scaffold complex was taken out and then washed with PBS. Hereafter, the cells on scaffolds were fixed with glutaraldehyde and dehydrated with gradient ethanol. Ultimately, the morphologies of the cells on the scaffolds were observed.

Fluorescence microscope (Olympus Co. Ltd., Tokyo, Japan) was adopted to observe the cell proliferation. The cell-scaffold complex was taken out from medium and washed with PBS for three times after culturing for 1, 3, and 5 days. Then, the cell-scaffold complex was stained with live/dead staining agent (PBS solution with $2 \mu \mathrm{M}$ calcein AM and $4 \mu \mathrm{M}$ EthD-1) and continuously incubated for another $30 \mathrm{~min}$. Finally, fluorescence microscope was selected to observe the living/dead cell morphologies.

The proliferation of MG-63 cell on scaffolds was quantitatively analyzed by the Cell Counting Kit-8 (CCK-8) assay. The cell-scaffold complex was gently washed with PBS and transferred into a new medium containing CCK- 8 reagent (Dojindo Laboratory, Kumamoto, Japan) after 1, 3, and 5 days of cultivation. $100 \mu \mathrm{L}$ of medium was moved to a 96 well plate after culture for another 2 hours, and the optical density was measured via a microplate reader (Beckman, USA) at $450 \mathrm{~nm}$.

Alkaline phosphatase (ALP), as a specific marker for early osteoblast differentiation, was observed by ALP staining. After 1, 3, and 5 days of cultivation, the cell-scaffold complex was gently rinsed with PBS and fixed with $4 \%$ paraformaldehyde for $20 \mathrm{~min}$. Subsequently, ALP staining was carried out using the ALP staining kit (Wako, Osaka, Japan), and images were photographed via a microscopy.

2.6. Statistical Analysis. One-way analysis of variance (ANOVA) was selected to evaluate the statistical significance. All data were presented as mean \pm standard deviations. The difference with $* p<0.05$ was recognized to be significant.

\section{Results and Discussion}

3.1. Microstructure. The digital photos and the microstructures of the represent scaffold with height of $10 \mathrm{~mm}$ and diameter of $10 \mathrm{~mm}$ are shown in Figure 2(a). Obviously, the scaffold displayed a well porous structure, which was consistent with the as-designed models. The pore size of the scaffold was approximately $500 \mu \mathrm{m}$. It had been reported that the minimum pore size of the bone scaffold should not be less than $100 \mu \mathrm{m}$ to ensure the nutrients transport and cells 


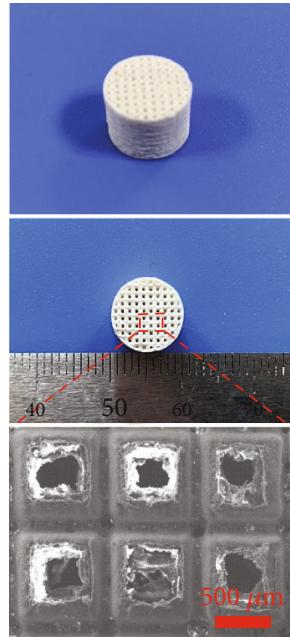

(a)

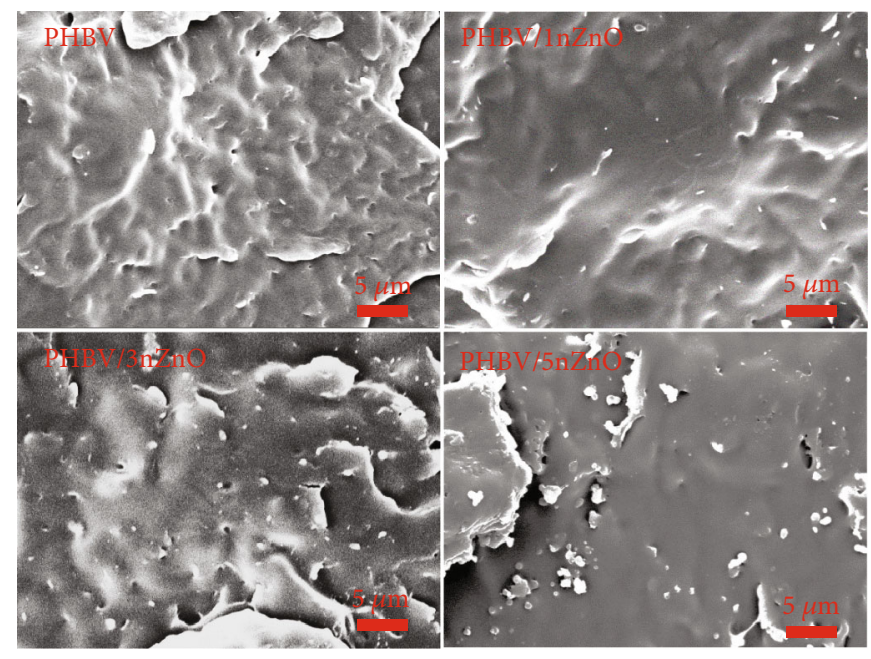

(b)

Figure 2: (a) The digital photos and the microstructures of the represent scaffold; (b) the cryofractured morphologies of PHBV and $\mathrm{PHBV} / \mathrm{nZnO}$ scaffolds.

growth [19]. Meanwhile, the mechanical performance of the scaffold would be deteriorated with the continuous ascent of the pore size, and the maximum pore size should not be higher than $1000 \mu \mathrm{m}$ [20]. It could be concluded that the pore size of the as-prepared scaffold was in the range of $100 \sim 1000 \mu \mathrm{m}$ and fulfilled the requirement of the bone scaffold. As reported in the literature, the dispersion state of the nanofillers in the polymer matrix had a significant influence on their comprehensive properties [21].

The cryofracture morphologies of the scaffolds are displayed in Figure 2(b). It could be obviously found that the composite scaffold presented a random and uniform nanofiller distribution with the content of $\mathrm{ZnO}$ lower than 3 wt.\%. However, the composite scaffold with 5 wt.\% $\mathrm{ZnO}$ presented poor nanofiller distribution, with small agglomeration composed of a few particles.

3.2. Thermal Behaviors. The thermal stabilities of the scaffold were measured by TG, and the results are shown in Figure 3(a). It could be seen from TG curves that all scaffolds exhibited a single decomposition stage. The initial decomposition temperature of PHBV scaffolds remarkably increased with the increase of $\mathrm{ZnO}$. For instance, the initial decomposition temperature of $\mathrm{PHBV} / 5 \mathrm{nZnO}$ scaffold increased from $261.2^{\circ} \mathrm{C}$ to $288.7^{\circ} \mathrm{C}$ in comparison with that of the PHBV scaffold. This improvement might be attributed to the interaction between the hydroxyl group of $\mathrm{ZnO}$ and the carbonyl of PHBV, which could form a barrier effect [22]. This barrier effect could prevent the transmission of the decomposition products. Furthermore, $\mathrm{ZnO}$ with excellent thermal conductivity could accelerate the heat dissipation in the composite and thereby enhance the thermal stability of the composite [23, 24]. It could also be found that the char residue of PHBV scaffold was gradually increased with increasing $\mathrm{ZnO}$ content, which was attributed to the relatively higher decomposition temperature of $\mathrm{ZnO}$ nanoparticles [25].
The melting and crystallization behaviors of the scaffolds are displayed in Figures 3(b) and 3(c). It could be seen from the melting curves that pure PHBV scaffold presented two obvious endothermic peaks located at $160.9^{\circ} \mathrm{C}$ and $170.6^{\circ} \mathrm{C}$, which were due to the melting of the initial crystals and the recrystallized crystals during the DSC heating process, respectively [26]. The endothermic peaks of the composite scaffolds decreased with the increase of $\mathrm{ZnO}$. The crystallization peak temperature of PHBV scaffolds gradually shifted to a higher temperature, which demonstrated that the nanofillers could efficiently promote the crystallization rate of the polymer (Figure 3(c)) [27]. In addition, the crystallization peak became more sharpened in the composite scaffolds, indicating that the nanofillers could efficiently accelerate the crystallization process of the polymer [28]. The relative crystallinity of the scaffolds could be calculated by the following equation:

$$
X_{\mathrm{c}}=\frac{\Delta H_{\mathrm{m}}}{\Delta H_{\mathrm{om}} \times w} \times 100 \% X_{\mathrm{c}}=\frac{\Delta H_{\mathrm{m}}}{\Delta H_{100} \times w} \times 100 \% \text {. }
$$

where $\Delta H_{\mathrm{m}}$ is the melting enthalpy of PHBV, $w$ is the mass fraction of PHBV in the composites, and $\Delta H_{m}^{o}$ is the theoretical enthalpy of PHBV (109 J/g) [29]. It could be found that there was a remarkable increase in the relative crystallinity of $\mathrm{PHBV} / 3 \mathrm{nZnO}$ composite scaffold from $38 \%$ to $64 \%$ in comparison with that of PHBV scaffolds, which might be attributed to the accelerated nucleation effect of $\mathrm{ZnO}$ nanoparticles. However, relative crystallinity of the composite scaffolds was decreased with the further increase of nanofillers. This might be due to the aggregation of the nanofillers, which hindered the mobility of the polymer chains during the crystallization process.

The XRD patterns of the scaffolds are shown in Figure 3(d). There was two obvious peaks located at $2 \theta$ of $13.4^{\circ}$ and $16.9^{\circ}$, being ascribed to the (020) and (110) crystalline planes of $\mathrm{PHBV}$, respectively [30]. The characteristic 


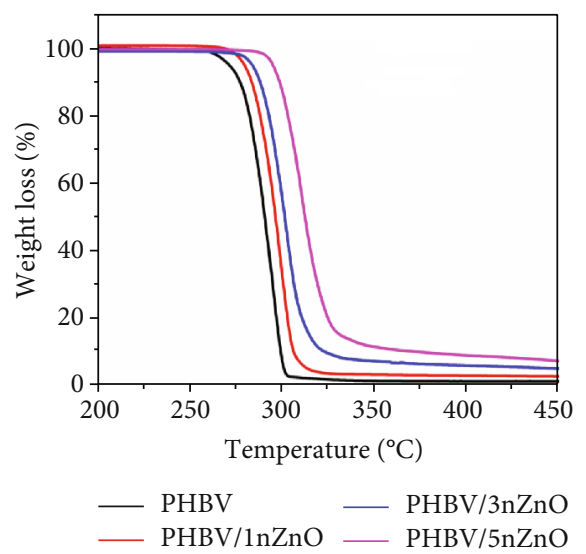

(a)

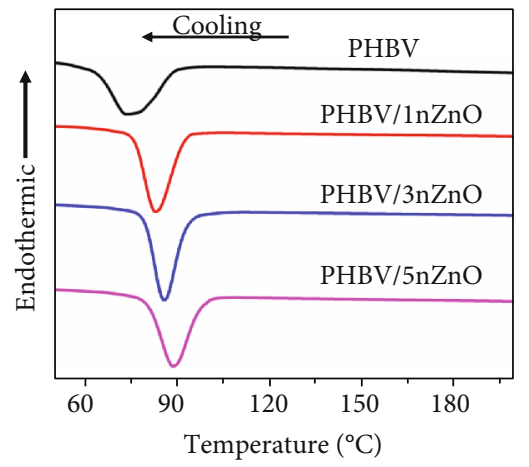

(c)

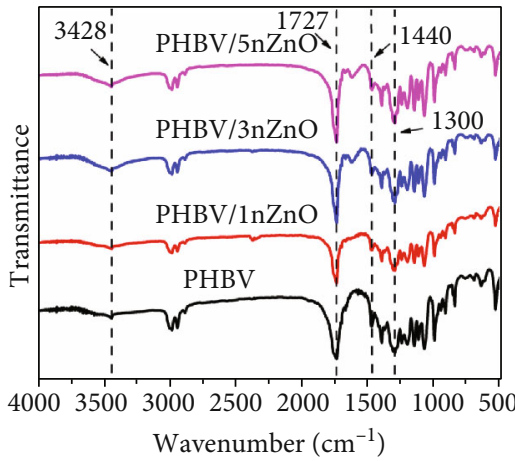

(e)

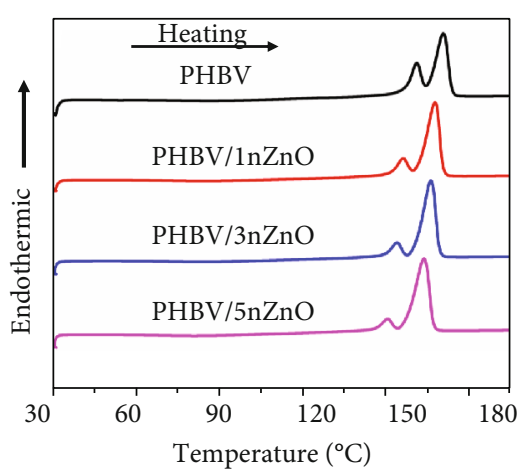

(b)

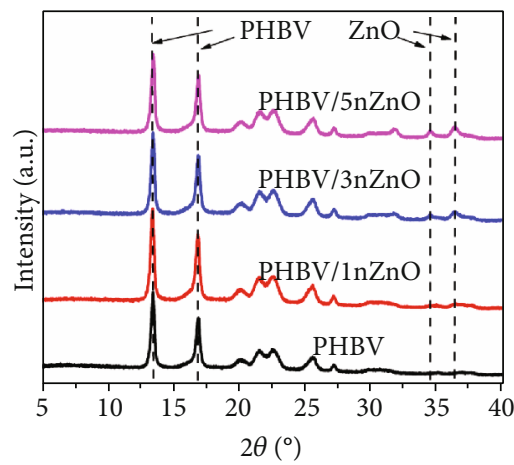

(d)

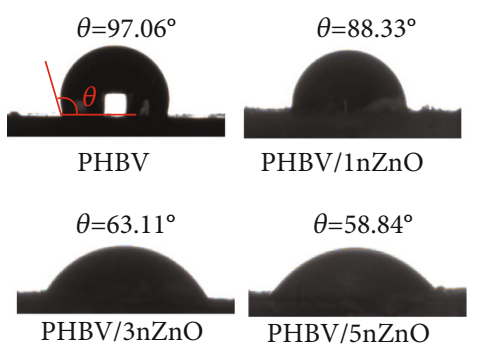

(f)

FIGURE 3: (a) TG curves, (b) DSC heating curves, (c) DSC cooling curves, (d) XRD patterns, (e) FTIR spectra, and (f) water contact angles of the scaffolds.

peaks located at $34.5^{\circ}$ and $36.1^{\circ}$ were ascribed to the (002) and (101) crystalline planes of $\mathrm{ZnO}$, respectively [31]. Compared with PHBV, the peak in the composite scaffold became narrower and more intense, which indicated that the nanofiller could efficiently promote the crystallization of PHBV, thereby resulting in the formation of smaller crystals. Moreover, there was no peak shift in the XRD patterns, which revealed that the SLS process did not destroy the crystal structures of the materials.

The functional groups of the scaffolds were characterized by FTIR, and the results are shown in Figure 3(e). Pure PHBV presented a strong peak situated at $1727 \mathrm{~cm}^{-1}$, which was assigned to the telescopic vibration of carbonyl group
[32]. The peaks situated at 1445 and $1300 \mathrm{~cm}^{-1}$, which were ascribed to the asymmetric bending vibration of $\mathrm{CH}_{3}$ and the in-plane bending vibration of $\mathrm{H}-\mathrm{C}-\mathrm{O}$ [33]. Once $\mathrm{ZnO}$ nanoparticles were incorporated, a broad band appeared at $3428 \mathrm{~cm}^{-1}$, being attributed to the stretching vibration of hydroxyl group on the nanoparticle surface [34]. It should be noted that the carbonyl band in the composite scaffolds became more intense and slightly shifted to lower wavenumber in comparison with that of pure PHBV, implying that there was a hydrogen bond interactions between the carbonyl group of PHBV and hydroxyl group of the nanoparticles [35].

The wettability of the scaffold had a significant influence on cell behaviors. It was established that the PHBV presented 


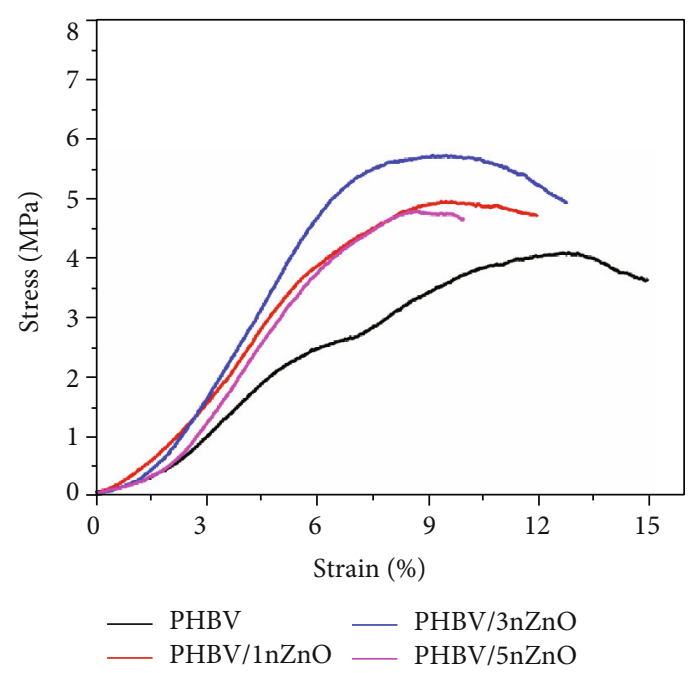

(a)

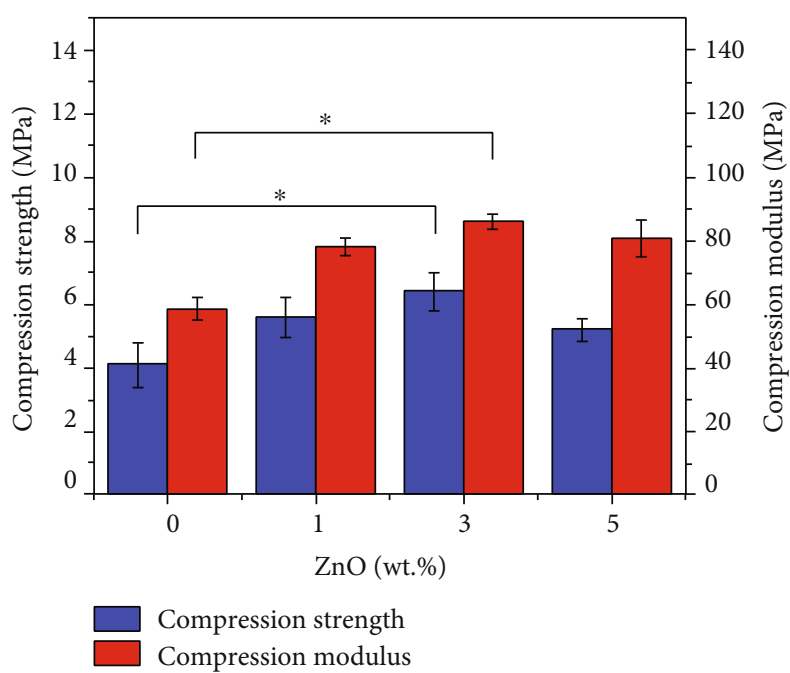

(b)

Figure 4: (a) The compression stress-strain curves, (b) compression strength and compression modulus of composite scaffold. $n=3$, $* p<0.05$.

inherent hydrophobic characteristic, which had limited its further application in bone defects treatment [36]. The water contact angles of the scaffold are shown in Figure 3(f). As expected, PHBV scaffold displayed a water contact angle of $97.06 \pm 1.33^{\circ}$. It was excited to found that the water contact angles of the composite scaffolds significantly decreased with the increase of $\mathrm{ZnO}$ content. For instance, the water contact angle of PHBV/5nZnO composite scaffold sharply decreased to $58.84 \pm 1.29^{\circ}$, which implied that the incorporation of $\mathrm{ZnO}$ could achieve a conversion of PHBV scaffold from hydrophobicity to hydrophilicity. This transformation might be attributed to a fact that the $\mathrm{ZnO}$ with hydroxyl groups was facilitated to the absorption of water molecules and thereby enhancing the hydrophilicity of the composite scaffolds [37].

3.3. Compressive Performance. The compressive performance of the scaffold plays a critical role in bearing different stresses and offering structural support to the bone tissues [38]. The compressive stress-strain curves were measured by mechanical testing equipment and are displayed in Figure 4(a). The compressive strength and compressive modulus of the composite scaffolds calculated from their stress-strain curves are presented in Figure 4(b). The compressive strength of the composite scaffolds firstly raised and then reduced with the increase of $\mathrm{ZnO}$ content. For instance, the compressive strength of $\mathrm{PHBV} / 3 \mathrm{nZnO}$ scaffold increased from $4.1 \pm 0.7$ to $6.4 \pm 0.6 \mathrm{MPa}$, which increased by $56 \%$ as compared with pure PHBV scaffold. This might mainly be ascribed to the combination of the increase in the crystallinity of PHBV and a uniform nanoparticle distribution. In detail, the uniformly distributed nanoparticles in the polymer could accelerate the orderly stacking of polymer chains, thus enhancing the crystallinity of the composites. The enhanced crystallinity could efficiently reduce the deformable space inside the composites, thereby enhancing their compressive strength. Meanwhile, the rigid nanoparticles could hinder the proliferation and development of cracks [39]. Moreover, the hydrogen bonding interaction between $\mathrm{PHBV}$ and $\mathrm{ZnO}$ might absorb a part of energy during the compressive process [40]. However, the compressive strength of the composite scaffold reduced with $\mathrm{ZnO}$ content further increasing to 5 wt.\%, which might be ascribed to the aggregation of $\mathrm{ZnO}$. Even so, the scaffold fabricated in this work still fulfilled the requirements for compressive strength of natural cancellous bone, which commonly exhibited a compressive strength of $1 \sim 10 \mathrm{MPa}$ [41]. Furthermore, it could be found that the compressive modulus of the composite scaffolds improved with the continuously increasing of $\mathrm{ZnO}$ content. For example, the compressive modulus of $\mathrm{PHBV} / 3 \mathrm{nZnO}$ scaffold increased from $58.6 \pm 3.36$ to $88.8 \pm 5.56 \mathrm{MPa}$, which increased by $51.5 \%$ in comparison with that of pure PHBV scaffold. This might be attributed to the relatively high modulus of $\mathrm{ZnO}$ nanoparticles, which was in agreement with the results as reported in the literature [42].

3.4. Antibacterial Properties. Trauma infection was still a big challenge in bone repair, which required bone implants to have antibacterial activity $[43,44]$. The bacterial inhibition rates of the scaffolds with various $\mathrm{ZnO}$ contents for 1, 4, and 7 days are shown in Figure 5(a). Obviously, the bacterial inhibition rates of the scaffolds increased with the extension of days. The bacterial morphologies on the various scaffolds for 7 days are shown in Figures 5(b). Obviously, several rod-like bacteria were adhered and connected to each other on the surface of PHBV scaffold. On the contrary, the number of adhered bacteria was greatly decreased with the introduction of $\mathrm{ZnO}$. More interestingly, the shapes of bacteria became distorted and shriveled, which implied that the cellular structure was damaged. It has been reported that the concentration of $\mathrm{Zn}^{2+}$ within $3 \mathrm{mg} / \mathrm{L}$ shows no cytotoxicity to normal cells [45]. In this work, the $\mathrm{Zn}^{2+}$ releases concentrations of scaffold in the deionized water for 1,4 , and 7 days were measured by ICP-OES, and the corresponding results is displayed in Figure 5(c). All scaffolds showed a slow 


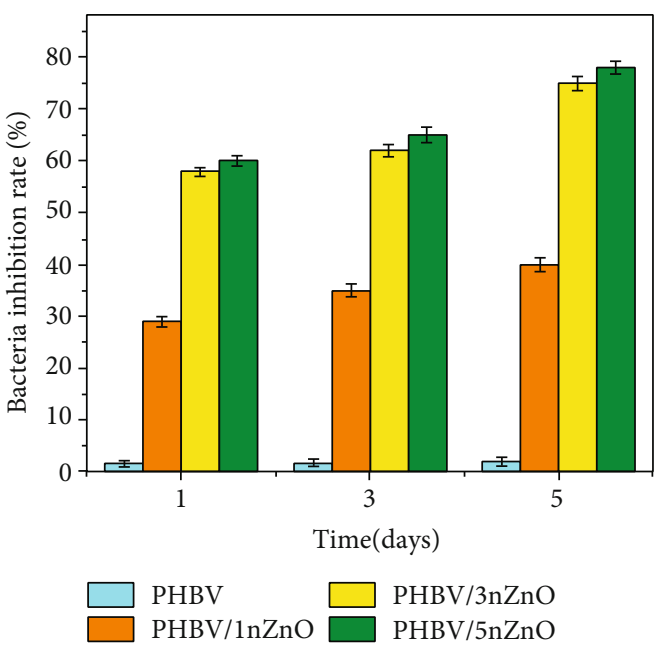

(a)

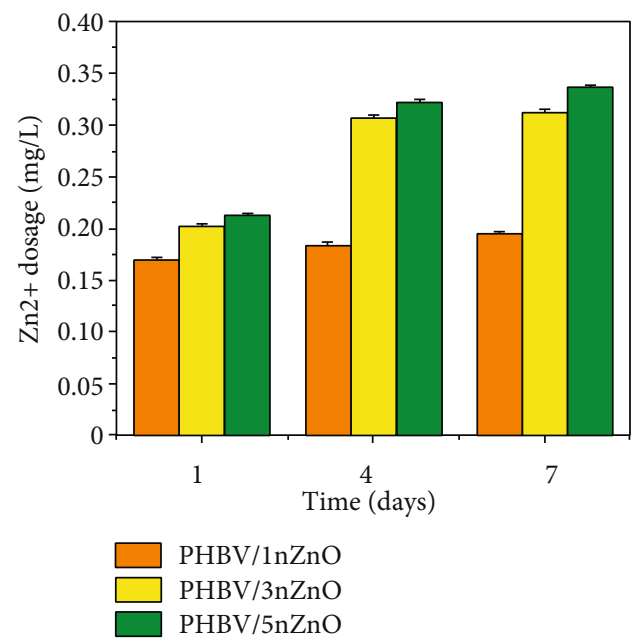

(c)

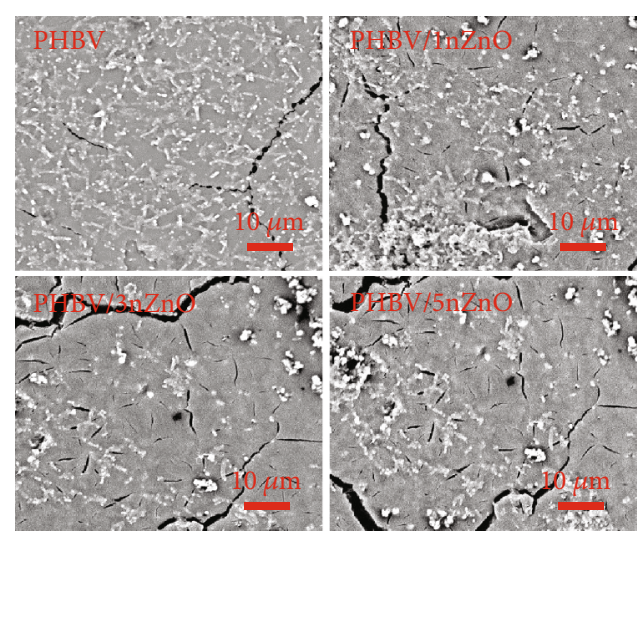

(b)

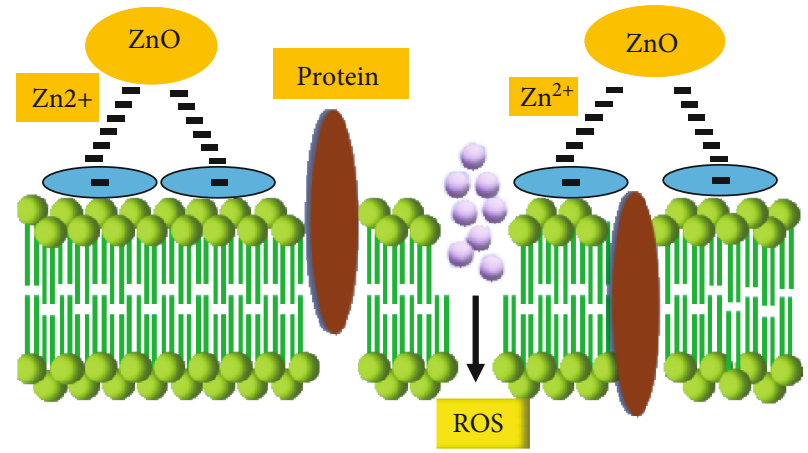

(d)

FIGURE 5: (a) Bacterial inhibition rate of E. coli cultured in medium containing various scaffolds for 1, 4, and 7 days; (b) bacterial morphology on the scaffolds; (c) $\mathrm{Zn}^{2+}$ release of the scaffolds after immersion in deionized water for different time; (d) a schematic for the antibacterial mechanism.

and sustained $\mathrm{Zn}^{2+}$ release throughout the whole process. The maximum released $\mathrm{Zn}^{2+}$ concentration of the scaffold was $0.3364 \pm 0.0024 \mathrm{mg} / \mathrm{L}$, which was much lower than $3 \mathrm{mg} / \mathrm{L}$. Therefore, the scaffolds have no negative effect on the normal function of cell.

Several antibacterial mechanisms had been proposed to interpret the antibacterial activity of $\mathrm{ZnO}$ nanoparticles, as shown in Figure 5(d). Briefly, it mainly consisted of the release of $\mathrm{Zn}^{2+}$; the mechanical damage of the cell membranes resulted from penetration of the nanoparticles, and the generation of reactive oxygen species [46]. In this work, the average size of $\mathrm{ZnO}$ was $50 \mathrm{~nm}$, which was unlikely to penetrate into the cell wall to destroy the bacteria [47]. Moreover, the production of reactive oxygen species by $\mathrm{ZnO}$ should be in the irradiation of ultraviolet light [48]. Therefore, the release of $\mathrm{Zn}^{2+}$ might be a potential reason for the antibacterial activity of the scaffolds. Pasquet et al.
[49] demonstrated that $\mathrm{Zn}^{2+}$ could absorb on the negatively charged bacterial wall by electrostatic interactions and thereby destroying the normal structure and function of the bacterial membrane, as well as interfering with protein metabolism and genetic expression of bacteria.

3.5. Cell Response. The cell response of the scaffolds plays a vital in bone repair $[50,51]$. Considering the mechanical and antibacterial properties of the scaffolds, the $\mathrm{PHBV} / 3 \mathrm{nZnO}$ scaffold was selected to further explore its cell behaviors. Cell adhesion was a prerequisite for a series of reactions such as migration, proliferation, and differentiation [52]. The SEM images of MG63 cells on PHBV and PHBV/3nZnO scaffolds for 1,3 , and 5 days are displayed in Figure 6. Apparently, the spread area of the cell on the scaffolds increased with the prolongation of time. It could be found that the cell on PHBV scaffold presented an ellipse shape in the whole culture 


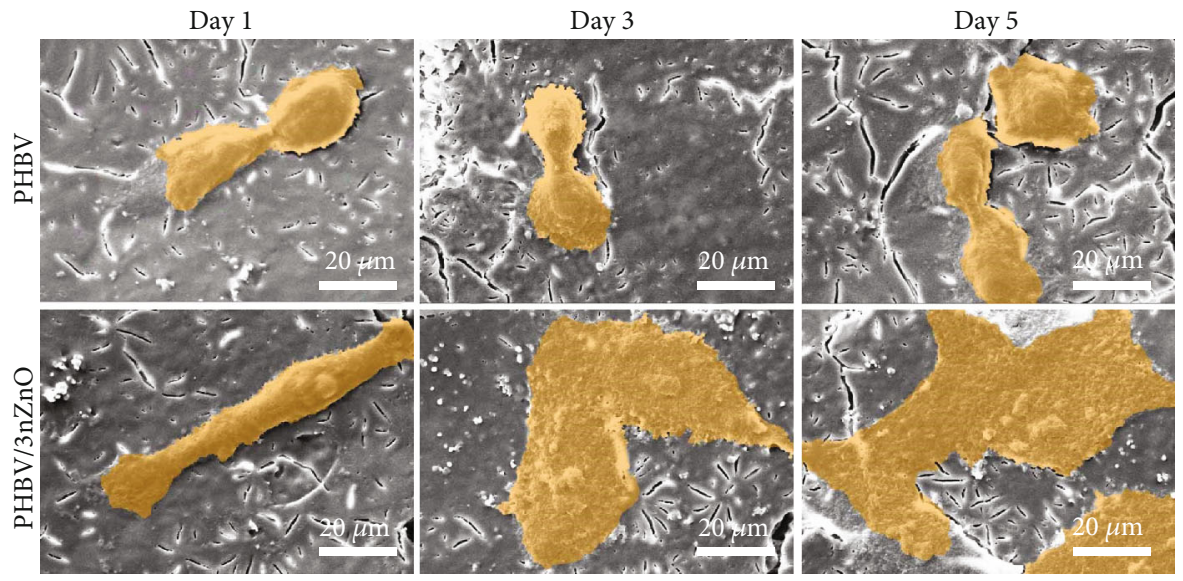

FIGURE 6: SEM images regarding the cell adhesion behavior on PHBV and PHBV/3nZnO scaffolds for 1,3 , and 5 days of culture.
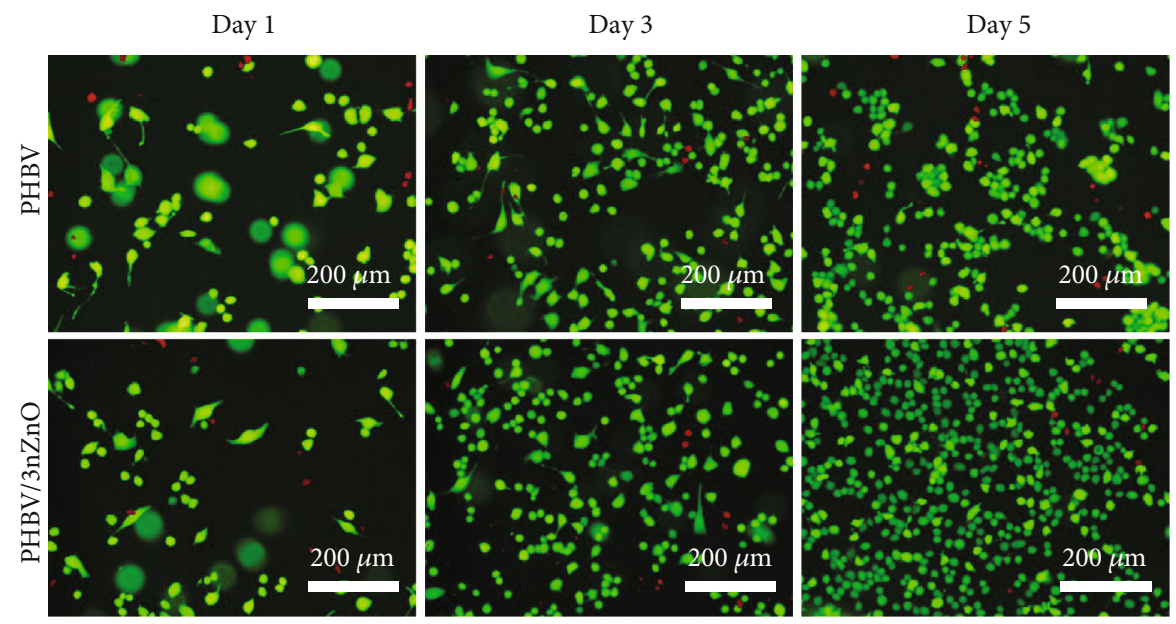

(a)

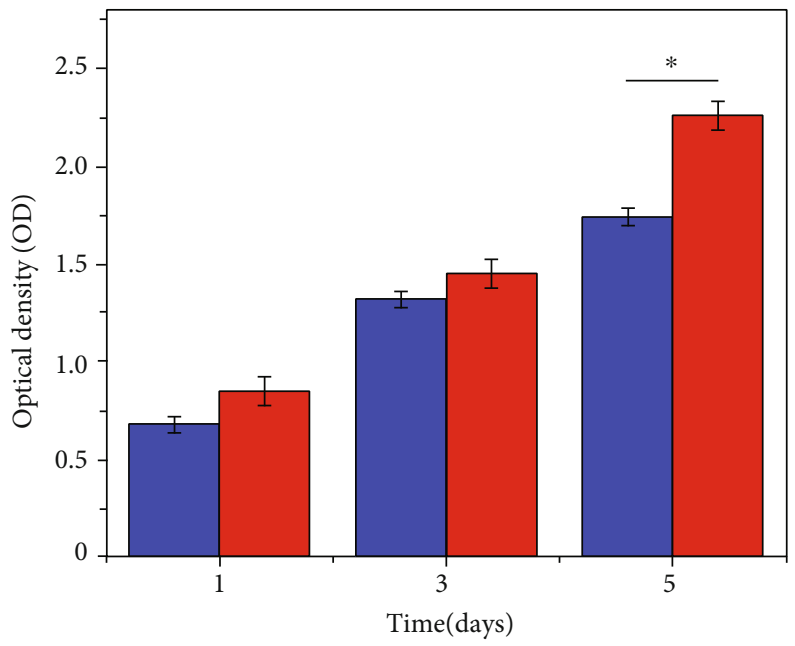

Compression strength

Compression modulus

(b)

FIGURE 7: (a) Fluorescence images and (b) optical density of cells on PHBV and PHBV/3nZnO scaffolds after 1, 3, and 5 days of culture. $n=3$, $* p<0.05$. 

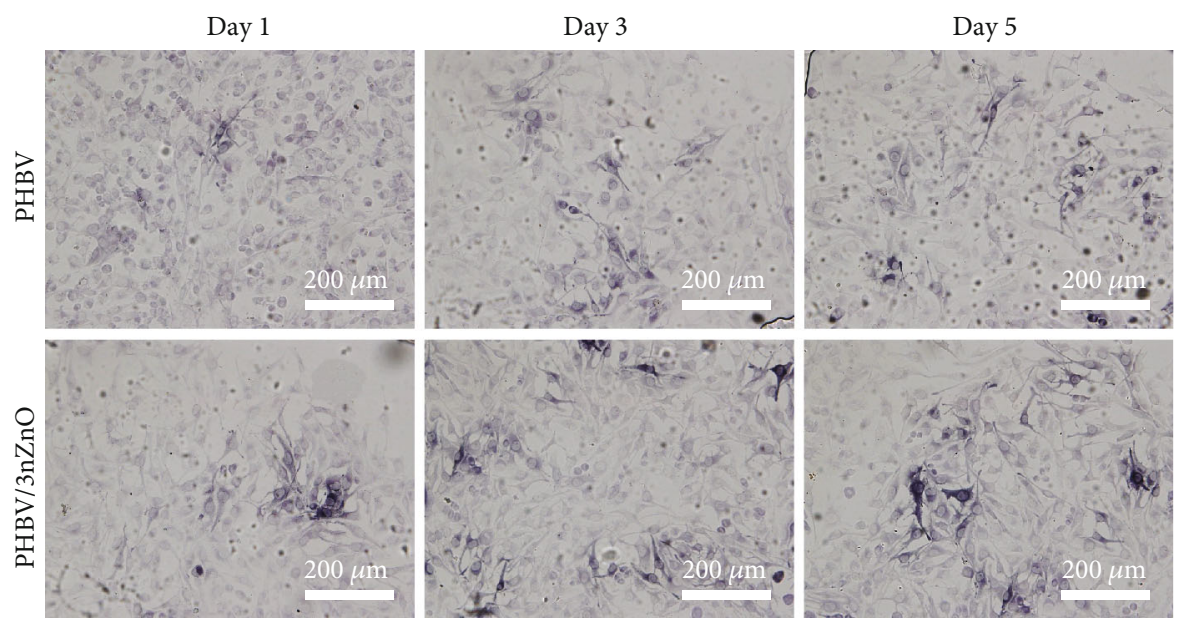

FIGURE 8: ALP staining images of MG-63 cells on PHBV and PHBV/3nZnO scaffolds after 1, 3, and 5 days of culture.

periods, which was attributed to the hydrophobic properties of the scaffolds [53]. Compared with the PHBV scaffold, the cells on PHBV/3nZnO displayed a flat and stretched shape after 1 day of culture. With the increase of culturing time, more adhered cells with longer filopodia attachment were observed on the PHBV/3nZnO scaffold, indicating its positive effect on cell adhesion and spreading.

Fluorescence test was performed to further explored the proliferation of cells on PHBV and PHBV/3nZnO scaffolds and the corresponding fluorescence images for 1,3 , and 5 days of cultures are shown in Figure 7(a). Live cells are stained in green, whereas dead cells are stained in red. Clearly, after 1-day culture, the cells on scaffold presented a healthy and normal polygonal shape, without obvious dead cells, which indicated that these scaffolds provided favorable survival environment. Meanwhile, the cell numbers increased significantly after 3 and 5 days of culture. The cell proliferation was quantitatively described by CCK- 8 measurements, and the results are displayed in Figure 7(b). The optical density of PHBV and PHBV/3nZnO scaffolds displayed a significant difference after 5-day culture, implying that the $\mathrm{ZnO}$ could promote the cell proliferation.

Alkaline phosphatase (ALP) activity, as a typical marker, was widely accepted to reflect the early differentiation of osteoblasts $[54,55]$. The staining images of MG-63 cell on PHBV and PHBV/3nZnO scaffolds after 1,3 , and 5 days of culture are presented in Figure 8. The ALP-positive areas of the cells increased with the increase of culture time. Moreover, the MG-63 cells seeded on PHBV/3nZnO scaffolds displayed a more significant ALP staining than that of PHBV scaffolds at the same culture time, which indicated that the $\mathrm{PHBV} / 3 \mathrm{nZnO}$ scaffolds could promote the cell differentiation. Combining with CCK-8 and live/dead staining assays, it was indicated that $\mathrm{PHBV} / 3 \mathrm{nZnO}$ scaffold was more conducive to cell adhesion, growth, and differentiation. The released $\mathrm{Zn}^{2+}$ could participate in the modulation of cellular signaling transduction [56]. Meanwhile, the $\mathrm{Zn}^{2+}$ could regulate the interaction between signal peptides and extramembrane receptors, thereby improving the cell behaviors [57]. In addition, $\mathrm{Zn}$ is an important trace element, which widely involves in synthesis of several nucleic acid and protein [58].

\section{Conclusion}

In this study, $\mathrm{ZnO}$ nanoparticles were incorporated into PHBV scaffolds fabricated by the SLS technique, aiming at improving their mechanical properties and antibacterial activities. The results indicated that a scaffold with $3 \mathrm{wt} . \% \mathrm{ZnO}$ exhibited a uniform dispersion and simultaneously could provide a nucleating site for the orderly stacking of PHBV chains. The relative crystallinity of $\mathrm{PHBV} / 3 \mathrm{nZnO}$ scaffold remarkably increased from $38 \%$ to $64 \%$ in comparison with that of pure PHBV scaffold. The improved crystallinity could effectively enhance the compression strength and modulus of the scaffold by $56 \%$ and $51.5 \%$, respectively. Moreover, $\mathrm{Zn}^{2+}$ released by the scaffolds could efficiently inhibit the growth of E. coli and promote the cell behaviors in terms of cell proliferation and differentiation. All these positive results confirmed that the scaffold was one potential bone scaffold material.

\section{Data Availability}

The case data used to support the findings of this study are included within the article.

\section{Conflicts of Interest}

The authors declare that they have no conflicts of interest.

\section{Acknowledgments}

This study was supported by the following funds: (1) The Natural Science Foundation of China (51935014, 51905553, 81871494, 81871498, and 51705540); (2) Hunan Provincial Natural Science Foundation of China (2019JJ50774, 2018 JJ3671, and 2019JJ50588); (3) The Provincial Key R\&D Projects of Jiangxi (20201BBE51012); (4) JiangXi Provincial Natural Science Foundation of China (20192ACB20005); (5) Guangdong Province Higher Vocational Colleges \& Schools Pearl River Scholar Funded Scheme (2018); (6) The Project of Hunan Provincial Science and Technology Plan (2017 RS3008), (7) Shenzhen Science and Technology Plan Project (JCYJ20170817112445033), (8) Innovation Team Project on 
University of Guangdong Province (2018GKCXTD001), and (9) Technology Innovation Platform Project of Shenzhen Institute of Information Technology 2020 (PT2020E002).

\section{References}

[1] X. Ye, L. Li, Z. Lin et al., "Integrating 3D-printed PHBV/calcium sulfate hemihydrate scaffold and chitosan hydrogel for enhanced osteogenic property," Carbohydrate Polymers, vol. 202, pp. 106-114, 2018.

[2] C. Shuai, G. Liu, Y. Yang et al., "A strawberry-like Agdecorated barium titanate enhances piezoelectric and antibacterial activities of polymer scaffold," Nano Energy, vol. 74, p. 104825, 2020.

[3] L. Zhang, G. Yang, B. N. Johnson, and X. Jia, "Three-dimensional (3D) printed scaffold and material selection for bone repair," Acta Biomaterialia, vol. 84, pp. 16-33, 2019.

[4] M. Kouhi, M. Fathi, M. P. Prabhakaran, M. Shamanian, and S. Ramakrishna, "Poly L lysine-modified PHBV based nanofibrous scaffolds for bone cell mineralization and osteogenic differentiation," Applied Surface Science, vol. 457, pp. 616-625, 2018.

[5] V. Jost and H.-C. Langowski, "Effect of different plasticisers on the mechanical and barrier properties of extruded cast PHBV films," European Polymer Journal, vol. 68, pp. 302-312, 2015.

[6] Z. Gu, R. Yang, J. Yang et al., "Dynamic Monte Carlo simulations of effects of nanoparticle on polymer crystallization in polymer solutions," Computational Materials Science, vol. 147, pp. 217-226, 2018.

[7] D. Jun, Z. Guomin, P. Mingzhu, Z. Leilei, L. Dagang, and Z. Rui, "Crystallization and mechanical properties of reinforced PHBV composites using melt compounding: effect of CNCs and CNFs," Carbohydrate Polymers, vol. 168, pp. 255262, 2017.

[8] M. Kouhi, M. P. Prabhakaran, M. Shamanian, M. Fathi, M. Morshed, and S. Ramakrishna, "Electrospun PHBV nanofibers containing HA and bredigite nanoparticles: fabrication, characterization and evaluation of mechanical properties and bioactivity," Composites Science and Technology, vol. 121, pp. 115-122, 2015.

[9] H. Zhang, H.-Y. Yu, C. Wang, and J. Yao, "Effect of silver contents in cellulose nanocrystal/silver nanohybrids on PHBV crystallization and property improvements," Carbohydrate Polymers, vol. 173, pp. 7-16, 2017.

[10] P. K. Mishra, H. Mishra, A. Ekielski, S. Talegaonkar, and B. Vaidya, "Zinc oxide nanoparticles: a promising nanomaterial for biomedical applications," Drug Discovery Today, vol. 22, no. 12, pp. 1825-1834, 2017.

[11] Y.-W. Wang, A. Cao, Y. Jiang et al., "Superior antibacterial activity of zinc oxide/graphene oxide composites originating from high zinc concentration localized around bacteria," ACS Applied Materials \& Interfaces, vol. 6, no. 4, pp. 27912798, 2014.

[12] Y. Li, W. Zhang, J. Niu, and Y. Chen, "Mechanism of photogenerated reactive oxygen species and correlation with the antibacterial properties of engineered metal-oxide nanoparticles," ACS Nano, vol. 6, no. 6, pp. 5164-5173, 2012.

[13] N. Padmavathy and R. Vijayaraghavan, "Enhanced bioactivity of $\mathrm{ZnO}$ nanoparticles-an antimicrobial study," Science and Technology of Advanced Materials, vol. 9, no. 3, article 035004, 2016.
[14] D. Bian, W. Zhou, J. Deng et al., "Development of magnesiumbased biodegradable metals with dietary trace element germanium as orthopaedic implant applications," Acta Biomaterialia, vol. 64, pp. 421-436, 2017.

[15] P. Deb, A. B. Deoghare, A. Borah, E. Barua, and S. Das Lala, "Scaffold development using biomaterials: a review," Materials Today: Proceedings, vol. 5, no. 5, pp. 12909-12919, 2018.

[16] C. Shuai, S. Li, W. Yang, Y. Yang, Y. Deng, and C. Gao, " $\mathrm{MnO}_{2}$ catalysis of oxygen reduction to accelerate the degradation of Fe-C composites for biomedical applications," Corrosion Science, vol. 170, article 108679, 2020.

[17] Y. Yang, C. Lu, S. Peng et al., "Laser additive manufacturing of Mg-based composite with improved degradation behaviour," Virtual and Physical Prototyping, vol. 15, pp. 278-293, 2020.

[18] G. Wang, C. He, W. Yang et al., "Surface-modified graphene oxide with compatible interface enhances Poly-L-Lactic acid bone scaffold," Journal of Nanomaterials, vol. 2020, Article ID 5634096, 11 pages, 2020.

[19] K. Zhang, Y. Fan, N. Dunne, and X. Li, "Effect of microporosity on scaffolds for bone tissue engineering," Regenerative Biomaterials, vol. 5, no. 2, pp. 115-124, 2018.

[20] S. Tarafder, V. K. Balla, N. M. Davies, A. Bandyopadhyay, and S. Bose, "Microwave-sintered 3D printed tricalcium phosphate scaffolds for bone tissue engineering," Journal of Tissue Engineering and Regenerative Medicine, vol. 7, no. 8, pp. 631-641, 2013.

[21] W. Noohom, K. S. Jack, D. Martin, and M. Trau, "Understanding the roles of nanoparticle dispersion and polymer crystallinity in controlling the mechanical properties of $\mathrm{HA} / \mathrm{PHBV}$ nanocomposites," Biomedical Materials, vol. 4, no. 1, article 015003, 2008.

[22] P. Li, Y. Zheng, M. Li et al., "Enhanced flame-retardant property of epoxy composites filled with solvent-free and liquidlike graphene organic hybrid material decorated by zinc hydroxystannate boxes," Composites Part A: Applied Science and Manufacturing, vol. 81, pp. 172-181, 2016.

[23] F.-Y. Yuan, H.-B. Zhang, X. Li, X.-Z. Li, and Z.-Z. Yu, "Synergistic effect of boron nitride flakes and tetrapod-shaped $\mathrm{ZnO}$ whiskers on the thermal conductivity of electrically insulating phenol formaldehyde composites," Composites Part A: Applied Science and Manufacturing, vol. 53, pp. 137-144, 2013.

[24] L. Fang, W. Wu, X. Huang, J. He, and P. Jiang, "Hydrangealike zinc oxide superstructures for ferroelectric polymer composites with high thermal conductivity and high dielectric constant," Composites Science and Technology, vol. 107, pp. 67-74, 2015.

[25] H. Wang, Z. Lu, D. Lu, C. Li, P. Fang, and X. Wang, "The synthesis of $\mathrm{Cu}$ /plate-like $\mathrm{ZnO}$ nanostructures and their selfassembly mechanism," Solid State Sciences, vol. 55, pp. 6976, 2016.

[26] E. B. C. Santos, J. J. P. Barros, D. A. de Moura, C. G. Moreno, F. de Carvalho Fim, and L. B. da Silva, "Rheological and thermal behavior of $\mathrm{PHB} /$ piassava fiber residue-based green composites modified with warm water," Journal of Materials Research and Technology, vol. 8, no. 1, pp. 531-540, 2019.

[27] I. Kaygusuz and C. Kaynak, "Influences of halloysite nanotubes on crystallisation behaviour of polylactide," Plastics, Rubber and Composites, vol. 44, no. 2, pp. 41-49, 2015.

[28] Q. Xing, R. Li, X. Dong et al., "Enhanced crystallization rate of poly (l-lactide) mediated by a hydrazide compound: 
nucleating mechanism study," Macromolecular Chemistry and Physics, vol. 216, no. 10, pp. 1134-1145, 2015.

[29] J. Castro-Mayorga, M. Fabra, and J. Lagaron, "Stabilized nanosilver based antimicrobial poly(3-hydroxybutyrate-co-3hydroxyvalerate) nanocomposites of interest in active food packaging," Innovative Food Science \& Emerging Technologies, vol. 33, pp. 524-533, 2016.

[30] L. Wei, N. M. Stark, and A. G. McDonald, "Interfacial improvements in biocomposites based on poly (3-hydroxybutyrate) and poly (3-hydroxybutyrate-co-3-hydroxyvalerate) bioplastics reinforced and grafted with $\alpha$-cellulose fibers," Green Chemistry, vol. 17, no. 10, pp. 4800-4814, 2015.

[31] M. Shaban, M. Zayed, and H. Hamdy, "Nanostructured ZnO thin films for self-cleaning applications," RSC Advances, vol. 7, no. 2, pp. 617-631, 2017.

[32] H.-Y. Yu and J.-M. Yao, "Reinforcing properties of bacterial polyester with different cellulose nanocrystals via modulating hydrogen bonds," Composites Science and Technology, vol. 136, pp. 53-60, 2016.

[33] A. Aramvash, S. Hajizadeh-Turchi, F. Moazzeni-zavareh, N. Gholami-Banadkuki, N. Malek-sabet, and Z. Akbari-Shahabi, "Effective enhancement of hydroxyvalerate content of PHBV in Cupriavidus necator and its characterization," International Journal of Biological Macromolecules, vol. 87, pp. 397-404, 2016.

[34] R. Saravanan, V. Gupta, T. Prakash, V. Narayanan, and A. Stephen, "Synthesis, characterization and photocatalytic activity of novel $\mathrm{Hg}$ doped $\mathrm{ZnO}$ nanorods prepared by thermal decomposition method," Journal of Molecular Liquids, vol. 178, pp. 88-93, 2013.

[35] Z. Yu, M. R. Kumar, D. Sun, L. Wang, and R. Hong, "Large scale production of hexagonal $\mathrm{ZnO}$ nanoparticles using PVP as a surfactant," Materials Letters, vol. 166, pp. 284-287, 2016.

[36] I. Unalan, O. Colpankan, A. Z. Albayrak, C. Gorgun, and A. S. Urkmez, "Biocompatibility of plasma-treated poly (3-hydroxybutyrate-co-3-hydroxyvalerate) nanofiber mats modified by silk fibroin for bone tissue regeneration," Materials Science and Engineering: C, vol. 68, pp. 842-850, 2016.

[37] Y. Qing, C. Yang, C. Hu, Y. Zheng, and C. Liu, "A facile method to prepare superhydrophobic fluorinated polysiloxane/ZnO nanocomposite coatings with corrosion resistance," Applied Surface Science, vol. 326, pp. 48-54, 2015.

[38] C. Shuai, L. Yu, P. Feng, C. Gao, and S. Peng, "Interfacial reinforcement in bioceramic/biopolymer composite bone scaffold: the role of coupling agent," Colloids and Surfaces B: Biointerfaces, vol. 193, article 111083, 2020.

[39] A. Ashori, M. Jonoobi, N. Ayrilmis, A. Shahreki, and M. A. Fashapoyeh, "Preparation and characterization of polyhydroxybutyrate-co-valerate (PHBV) as green composites using nano reinforcements," International Journal of Biological Macromolecules, vol. 136, pp. 1119-1124, 2019.

[40] W. Mook Choi, T. Wan Kim, O. Ok Park, Y. Keun Chang, and J. Woo Lee, "Preparation and characterization of poly (hydroxybutyrate-co-hydroxyvalerate)-organoclay nanocomposites," Journal of Applied Polymer Science, vol. 90, no. 2, pp. 525-529, 2003.

[41] S.-I. Roohani-Esfahani, P. Newman, and H. Zreiqat, "Design and fabrication of $3 \mathrm{D}$ printed scaffolds with a mechanical strength comparable to cortical bone to repair large bone defects," Scientific Reports, vol. 6, no. 1, article 19468, 2016.
[42] C. Shuai, B. Wang, S. Bin, S. Peng, and C. Gao, "Interfacial strengthening by reduced graphene oxide coated with $\mathrm{MgO}$ in biodegradable Mg composites," Materials \& Design, vol. 191, p. 108612, 2020.

[43] A. Bari, N. Bloise, S. Fiorilli et al., "Copper-containing mesoporous bioactive glass nanoparticles as multifunctional agent for bone regeneration," Acta Biomaterialia, vol. 55, pp. 493504, 2017.

[44] E. Barua, A. B. Deoghare, S. Chatterjee, and P. Sapkal, "Effect of $\mathrm{ZnO}$ reinforcement on the compressive properties, in vitro bioactivity, biodegradability and cytocompatibility of bone scaffold developed from bovine bone-derived HAp and PMMA," Ceramics International, vol. 45, no. 16, pp. 2033120345, 2019.

[45] G. Jin, H. Cao, Y. Qiao, F. Meng, H. Zhu, and X. Liu, “Osteogenic activity and antibacterial effect of zinc ion implanted titanium," Colloids and Surfaces B: Biointerfaces, vol. 117, pp. 158-165, 2014.

[46] J. Li, L. Tan, X. Liu et al., "Balancing bacteria-osteoblast competition through selective physical puncture and biofunctionalization of $\mathrm{ZnO} /$ polydopamine/arginine-glycine-aspartic acidcysteine nanorods," ACS Nano, vol. 11, no. 11, pp. 1125011263, 2017.

[47] R. Javed, M. Usman, S. Tabassum, and M. Zia, "Effect of capping agents: structural, optical and biological properties of ZnO nanoparticles," Applied Surface Science, vol. 386, pp. 319-326, 2016.

[48] J. Podporska-Carroll, A. Myles, B. Quilty et al., "Antibacterial properties of F-doped $\mathrm{ZnO}$ visible light photocatalyst," Journal of Hazardous Materials, vol. 324, pp. 39-47, 2017.

[49] J. Pasquet, Y. Chevalier, E. Couval, D. Bouvier, and M.A. Bolzinger, "Zinc oxide as a new antimicrobial preservative of topical products: interactions with common formulation ingredients," International Journal of Pharmaceutics, vol. 479, no. 1, pp. 88-95, 2015.

[50] C. Shuai, B. Wang, S. Bin, S. Peng, and C. Gao, “TiO2 induced in situ reaction in graphene oxide reinforced AZ61 biocomposites to enhance the interfacial bonding," ACS Applied Materials \& Interfaces, vol. 12, no. 20, pp. 23464-23473, 2020.

[51] C. Shuai, Y. Cheng, W. Yang et al., "Magnetically actuated bone scaffold: microstructure, cell response and osteogenesis," Composites Part B: Engineering, vol. 192, p. 107986, 2020.

[52] L. Cao, W. Liu, Y. Zhong et al., "Linc02349 promotes osteogenesis of human umbilical cord-derived stem cells by acting as a competing endogenous RNA for miR-25-3p and miR33b-5p," Cell Proliferation, vol. 53, no. 5, article e12814, 2020.

[53] X. Li, J. Wei, K. E. Aifantis et al., "Current investigations into magnetic nanoparticles for biomedical applications," Journal of Biomedical Materials Research Part A, vol. 104, no. 5, pp. 1285-1296, 2016.

[54] S. He, S. Yang, Y. Zhang et al., "LncRNA ODIR1 inhibits osteogenic differentiation of hUC-MSCs through the FBXO25/H2BK120ub/H3K4me3/OSX axis," Cell Death of Disease, vol. 10, no. 12, article 947, 2019.

[55] W. Yang, Y. Zhong, C. He et al., "Electrostatic self-assembly of pFe3O4 nanoparticles on graphene oxide: a co-dispersed nanosystem reinforces PLLA scaffolds," Journal of Advanced Research, vol. 24, pp. 191-203, 2020.

[56] G. Zhang, Y. Zhao, B. Peng et al., "A fluorogenic probe based on chelation-hydrolysis-enhancement mechanism for 
visualizing Zn 2+ in Parkinson's disease models," Journal of Materials Chemistry B, vol. 7, no. 14, pp. 2252-2260, 2019.

[57] Z. Zhang, H. Hao, Z. Tang et al., "Identification and characterization of a new alkaline thermolysin-like protease, BtsTLP1, from Bacillus thuringiensis Serovar Sichuansis strain MC28," Journal of Microbiology and Biotechnology, vol. 25, no. 8, pp. 1281-1290, 2015.

[58] A. Fallah, A. Mohammad-Hasani, and A. H. Colagar, "Zinc is an essential element for male fertility: a review of $\mathrm{Zn}$ roles in men's health, germination, sperm quality, and fertilization," Journal of Reproduction \& Infertility, vol. 19, no. 2, p. 69, 2018. 